\title{
Determining the Anchoring Strength of a Capillary Using Topological Defects
}

\author{
Randall D. Kamien ${ }^{\dagger}$ \\ Department of Physics and Astronomy, University of Pennsylvania, Philadelphia, PA 19104 \\ and \\ Thomas R. Powers ${ }^{\ddagger}$ \\ Department of Physics, University of Arizona, Tucson, AZ 85721
}

\begin{abstract}
We consider a smectic- $A^{*}$ in a capillary with surface anchoring that favors parallel alignment. If the bulk phase of the smectic is the standard twist-grain-boundary phase of chiral smectics, then there will be a critical radius below which the smectic will not have any topological defects. Above this radius a single screw dislocation in the center of the capillary will be favored. Along with surface anchoring, a magnetic field will also suppress the formation of a screw dislocation. In this note, we calculate the critical field at which a defect is energetically preferred as a function of the surface anchoring strength and the capillary radius. Experiments at a few different radii could thus determine the anchoring strength.
\end{abstract}

17 December 1996; Revised 1 February 1997

\footnotetext{
$\dagger$ email: kamien@lubensky.physics.upenn.edu

$\ddagger$ email: powers@davinci.physics.arizona.edu
} 
Boundary conditions play an essential role in liquid crystal physics and they cannot be taken with a cavalier attitude. Even if the boundary is very far away, surface effects in liquid crystals can be very important because of typically long-range, algebraic correlations in these soft materials. Indeed, in device applications one is often interested in the surface effects on bulk ordering: such effects are a key element in the twisted nematic display [1].

In this note we consider a smectic liquid crystal which, in bulk, would form a RennLubensky [2] twist-grain-boundary (TGB) phase [3]. We will show that if this smectic is confined to the classic capillary geometry [4,5] with the layer normals parallel to the capillary axis then for sufficiently small radii an undefected smectic- $A$ phase persists while for larger radii screw dislocations can enter as shown in figure 1. While this, in principle, can give a very clean determination of the anchoring strength $W$ it is rather impractical to do an experiment on a sequence of capillaries with small differences in their radial size. Instead, we show how the imposition of a magnetic field also suppresses defect formation. In this case, at fixed radii, the applied field may be scanned and the critical field may be determined. Doing this measurement at a few radii should make it possible to determine $W$.

We start with the free energy of a smectic confined to a region $\Omega$ in the $x y$-plane, and assume that the capillary is infinite in the $z$ direction. We further assume there is an infinite energy cost for the director to have a radial component at the surface. The appropriate free energy to quadratic order is then [6]

$$
\begin{aligned}
F= & F_{\text {bulk }}+F_{\text {surface }} \\
= & \int \mathrm{d} z \int_{\Omega} \mathrm{d}^{2} x\left\{\frac{B}{2}\left(\nabla_{\perp} u+\delta \mathbf{n}\right)^{2}+\frac{B_{z}}{2}\left(\partial_{z} u\right)^{2}+\frac{K_{1}}{2}\left(\nabla_{\perp} \cdot \delta \mathbf{n}\right)^{2}+\frac{K_{2}}{2}\left(\nabla_{\perp} \times \delta \mathbf{n}\right)^{2}\right. \\
& \left.\quad+\frac{K_{3}}{2}\left(\partial_{z} \delta \mathbf{n}\right)^{2}-\frac{\chi}{2}(\mathbf{H} \cdot \mathbf{n})^{2}\right\} \\
- & \int \mathrm{d} z\left\{\int_{\partial \Omega} \mathrm{d} \ell\left[K_{2} q_{0} \delta \mathbf{n} \cdot \mathbf{T}+\frac{K_{24}}{2} \mathbf{N} \cdot[\mathbf{n}(\nabla \cdot \mathbf{n})-(\mathbf{n} \cdot \nabla) \mathbf{n}]\right]-\int_{\partial_{+} \Omega} \mathrm{d} \ell\left[\frac{W}{2}\left(\mathbf{n}_{\phi}\right)^{2}\right]\right\} .
\end{aligned}
$$

$\partial \Omega$ denotes the total boundary of the smectic region, including any boundaries at defects, while $\partial_{+} \Omega$ includes only the boundary with the capillary; $\mathbf{N}$ is the (outward pointing) normal to the surface and $\mathbf{T}$ is the surface tangent perpendicular to $\hat{z}$. The smectic order parameter is $\psi=|\psi| e^{i 2 \pi(z+u) / a}$, and thus the phase of the mass-density wave is $u$. The molecular director is $\mathbf{n}=\hat{z} \sqrt{1-\delta n^{2}}+\delta \mathbf{n}$ with $\hat{z} \cdot \delta \mathbf{n}=0$. The $K_{i}$ are the Frank elastic constants, $B$ and $B_{z}$ are proportional to the smectic order parameter squared $|\psi|^{2}, K_{24}$ 
is the saddle-splay elastic constant [7], $q_{0}$ is the equilibrium cholesteric pitch, $\chi$ is the negative diamagnetic susceptibility, $\mathbf{H}=H \hat{z}$ is a magnetic field, and $W$ is the anchoring strength which we want to determine. In (1) we have used the fact that the chiral bulk term $K_{2} q_{0} \nabla_{\perp} \times \delta \mathbf{n}$ can be rewritten as a surface term. While we are not explicitly interested in the saddle-splay term, there is no way to remove it from the problem - when there is a surface it must be included [8,5]. It too serves to promote or hinder the formation of a central screw dislocation.

We note by symmetry along $\hat{z}$ that the problem can be reduced to a two-dimensional problem and thus the fields will have no $z$ dependence. The field configurations which minimize the bulk free energy satisfy the Euler-Lagrange equations:

$$
\begin{aligned}
& 0=\nabla_{\perp}^{2} u+\nabla_{\perp} \cdot \delta \mathbf{n} \\
& 0=B\left(\nabla_{\perp} u+\delta \mathbf{n}\right)+\chi H^{2} \delta \mathbf{n}-K_{1} \nabla_{\perp} \nabla_{\perp} \cdot \delta \mathbf{n}-K_{2}\left(\nabla_{\perp}^{2} \delta \mathbf{n}-\nabla_{\perp} \nabla_{\perp} \cdot \delta \mathbf{n}\right)
\end{aligned}
$$

The saddle-splay term can lead to a buckling instability toward a configuration with a nonzero divergence of $\delta \mathbf{n}$. In this note we will limit our attention to the effect of the anchoring term on defect formation; in our quadratic approximation the transverse distortions due to the defect decouple from the longitudinal distortions due to buckling. We will therefore assume $\nabla_{\perp} \cdot \delta \mathbf{n}=0$. It would be an interesting extension of this work to study the nonlinear theory to understand buckling and its interplay with defects (cf. [9]).

We write this constraint in cylindrical coördinates as

$$
\frac{1}{\rho} \partial_{\rho}\left(\rho \delta n_{\rho}\right)+\frac{1}{\rho} \partial_{\phi} \delta n_{\phi}=0
$$

Using rotational invariance, we can assume that the nematic field $\delta \mathbf{n}$ is independent of $\phi$. In this case (3) implies that $\rho \delta n_{\rho}$ is constant. Since $\delta n$ must be well defined everywhere, including $\rho=0$, the constant must be 0 . Therefore $\delta \mathbf{n}=\delta n_{\phi}(\rho) \hat{\phi}$.

To find the minimum energy solution we must take into account the boundary terms. The outer surface energy in (1) may be expanded up to quadratic order in $\delta \mathbf{n}$ :

$$
F_{\text {surface }}=\int \mathrm{d} z \int_{\partial_{+} \Omega} \mathrm{d} \ell\left[\delta n_{\phi}(R)\right]^{2}\left[\frac{W}{2}-\frac{K_{24}}{2 R}\right]-K_{2} q_{0} \int \mathrm{d} z \int_{\partial_{+} \Omega} \mathrm{d} \ell \delta n_{\phi}(R)
$$

where $R$ is the capillary radius. We work in the type II [6] limit in which the twist penetration depth $\lambda_{0}=\sqrt{K_{2} / B}$ is much bigger than the coherence length $\xi$; in this 
limit the contribution of the inner boundary terms turns out to be subleading. We may now calculate the minimum bulk energy for fixed $\delta n_{\phi}(R)$ and get an effective free energy $F\left(\delta n_{\phi}(R)\right)$ which we may finally minimize over the number $\delta n_{\phi}(R)$. At this point we identify the magnetic twist penetration depth $\lambda_{H}^{2}=K_{2} /\left(B+\chi H^{2}\right)$. We can solve (2) by introducing $\mathbf{Q}=\delta \mathbf{n}+\left(\lambda_{H}^{2} / \lambda_{0}^{2}\right) \nabla_{\perp} u[1]$ so that

$$
\nabla_{\perp}^{2} \mathbf{Q}-\frac{1}{\lambda_{H}^{2}} \mathbf{Q}=0
$$

Note that (2b) implies that $u$ is independent of $\rho$ and thus $\mathbf{Q}$ only has components in the $\hat{\phi}$ direction. In cylindrical coördinates (5) reduces to Bessel's modified equation with index $\nu=1$ and so

$$
Q_{\phi}(\rho)=C_{1} K_{1}\left(\rho / \lambda_{H}\right)+C_{2} I_{1}\left(\rho / \lambda_{H}\right)
$$

We determine $C_{1}$ by insisting that $\delta \mathbf{n}$ be regular at the origin. The origin is, in fact, the other boundary. In particular, we might consider the possibility that there is a defect at the center of the capillary. Recall that a screw dislocation is a layer configuration in which $u=a m \phi /(2 \pi)$ where $a$ is the layer spacing and $m$ is an integer. Note that since $u$ is independent of $\rho, u=a m \phi /(2 \pi)$ is the most general solution of the equations of equilibrium with $\nabla_{\perp} \cdot \delta \mathbf{n}=0$. This implies that $\nabla_{\perp} u=\hat{\phi} \frac{a m}{(2 \pi \rho)}$ and is thus singular at the origin. Since $I_{1}(0)=0$, we have, near $\rho=0$

$$
\rho \delta n_{\phi}(0)+\frac{\lambda_{H}^{2}}{\lambda_{0}^{2}} \frac{a m}{2 \pi}=C_{1} \lambda_{H}
$$

and so $C_{1}=a m \lambda_{H} /\left(2 \pi \lambda_{0}^{2}\right)$. Thus the only free parameter in the bulk free energy is $C_{2}$ which is determined by demanding $\delta n_{\phi}(R) \equiv \delta n_{0}$ :

$$
C_{2}=\frac{\delta n_{0}+a m \lambda_{H} /\left(2 \pi \lambda_{0}^{2}\right)\left[\left(\lambda_{H} / R\right)-K_{1}\left(R / \lambda_{H}\right)\right]}{I_{1}\left(R / \lambda_{H}\right)} .
$$

Taking the core size to be $\xi$ and working in the regime where $R \gg \lambda_{0}, \lambda_{H} \gg \xi$ we find that the bulk strain energy to leading order in $\lambda_{H} / R$ is

$$
\begin{aligned}
F_{\text {bulk }}\left(\delta n_{0}\right) / L & \approx m^{2} E_{\text {core }}+\chi H^{2} \frac{a^{2} m^{2} \lambda_{H}^{2}}{4 \pi \lambda_{0}^{2}} \ln (R / \xi)+K_{2} \frac{a^{2} m^{2} \lambda_{H}^{2}}{4 \pi \lambda_{0}^{4}} \ln \frac{\lambda_{H}}{\xi} \\
& +\delta n_{0}^{2} K_{2} \pi \frac{R}{\lambda_{H}}+\delta n_{0} K_{2} \frac{\lambda_{H} a m}{\lambda_{0}^{2}}
\end{aligned}
$$

where $m^{2} E_{\text {core }}$ is the energy cost per unit length of destroying the smectic order at the defect core. Note that the strain energy depends not only on the boundary value of 
$\delta \mathbf{n}=\delta n_{0} \hat{\phi}$, but also on the strength of the defect $m$. When minimizing, we must minimize over both variables. Choosing the minimum over $m$ will indicate whether or not there is a screw dislocation at all.

Adding this energy to the surface energy (4), we minimize over $\delta n_{0}$ to find the minimum energy $\widetilde{F}$. To determine the relative magnitudes of the terms, we estimate $K_{2} \approx$ $K_{24}=\mathcal{O}\left(\frac{k_{\mathrm{B}} T}{\xi}\right)$ and $a \approx \xi$. In NMR studies [10] it has been seen that $W / K_{24} \approx(28 \mathrm{~nm})^{-1}$ for non-chiral nematic liquid crystal in small capillaries in Nuclepore; we therefore expect that $K_{24} / R \ll W$ for radii in the range of $R \sim 10 \mu \mathrm{m}$. Thus we find a tipping of

$$
\delta n_{0} \approx \frac{K_{2} q_{0}}{W} \frac{1-\frac{\lambda_{H} m a}{2 \pi \lambda_{0}^{2} q_{0} R}}{1+\frac{K_{2}}{W \lambda_{H}}}
$$

For large $R$ the tipping angle at the capillary wall is independent of $R$ and $m$. This is because the director can lower the chiral energy by tipping at the wall even in the absence of a defect; the director will align with the layer normal at a distance of a penetration depth from the wall. Therefore we must subtract off the strain energy of the zero defect state to arrive at the dislocation energy

$$
\widetilde{F} / L \approx m^{2}\left\{E_{\text {core }}+\chi H^{2} \frac{a^{2} \lambda_{H}^{2}}{4 \pi \lambda_{0}^{2}} \ln \frac{R}{\xi}+K_{2} \frac{a^{2} \lambda_{H}^{2}}{4 \pi \lambda_{0}^{4}} \ln \frac{\lambda_{H}}{\xi}\right\}+m \frac{K_{2}^{2} a q_{0} \lambda_{H}}{\left(W+\frac{K_{2}}{\lambda_{H}}\right) \lambda_{0}^{2}}
$$

We have kept the subleading term $K_{2} /\left(W \lambda_{H}\right)$ so that our expressions have sensible large $H$ behaviour, e.g. $\delta n_{0} \rightarrow 0$, as $H \rightarrow \infty$, even though in this limit we leave the type II regime. Each term of (11) has a simple interpretation: the first three terms are the energy of a screw dislocation in a bulk sample subject to a magnetic field, and the last term is the usual chiral term of a bulk defect reduced by a factor involving the anchoring.

Note that if $q_{0}=0$ then the minimum energy is at $m=0$, in other words, no defect. It is easy to see that there are critical values of $H^{2}, R$ and $q_{0}$ for which the free energy will be minimized for $m \neq 0$. First we consider zero magnetic field. The sign of the Burgers vector is determined by the sign of $q_{0}$; we assume that $q_{0}>0$. The critical value of the chirality needed to get a defect with $m=-1$ in a very large capillary $(R \rightarrow \infty)$ is increased from its bulk value by the anchoring:

$$
q_{0 \mathrm{c}} \approx q_{0 \mathrm{c}, \mathrm{bulk}}\left(\frac{W \lambda_{0}}{K_{2}}+1\right)
$$

where

$$
q_{0 \mathrm{c}, \mathrm{bulk}}=\frac{m}{a}\left[\frac{E_{\mathrm{core}}}{K_{2}}+\frac{a^{2}}{4 \pi \lambda_{0}^{2}} \log \left(\lambda_{0} / \xi\right)\right]
$$


In general the critical chirality depends on the radius. Equivalently, there is a critical radius $R_{\mathrm{c}}$ depending on $q_{0}$ such that if $R<R_{\mathrm{c}}$, no defect will occur. Unless $q_{0}$ is very close to its critical value, this radius is typically the size of the penetration depth, in which case our expressions are not valid. We can, of course, compute $R_{\mathrm{c}}$ and its dependence on $W$ by dropping the assumption $R \gg \lambda_{0}$, but as noted above $R_{\mathrm{c}}$ is not so easy to determine experimentally.

Now consider subjecting the sample to a magnetic field. For a capillary with radius $R>R_{\mathrm{c}}$, there is a critical value $H_{\mathrm{c}}(R, W)$ of the field above which there will be no defect; $H_{\mathrm{c}}(R, W)$ is the number such that the right hand side of (11) is zero for $m \neq 0$. While an analytic expression for $H=H_{c}$ is complicated, we can easily plot $H_{c}(R, W)$ for a system in which the elastic constants are known. Thus a given liquid crystal system may be studied in only a few capillaries in order to determine which value of $W$ fits the observed formation of a defect. In fact, due to the logarithmic dependence of the magnetic strain energy on the radius, the critical field is a very weak function of $R$ so that just one measurement may suffice to determine $W$. In figure 2 we show $H_{c}$ vs. $R$ for various $W$, with $R$ ranging over typical capillary sizes of $10-100 \mu \mathrm{m} ; \bar{R} \equiv R / \lambda_{0}$, $\bar{H}^{2} \equiv \chi H^{2} / B, \bar{W} \equiv W \lambda_{0} / K_{2}$, and $q_{0}=11 . \mu \mathrm{m}^{-1} . \bar{W}$ ranges from 0.3 to 3.0. We have taken $\lambda_{0}=0.1 \mu \mathrm{m}, 4 \pi \lambda_{0}^{2} E_{\text {core }} /\left(a^{2} K_{2}\right)=1$, and $\lambda_{0} / \xi=10$. Note that the magnetic fields in the ranges we consider do not spoil the type II behavior since $\lambda_{H}>\xi$.

In summary we have proposed a set of experiments that could be performed on liquid crystals that form TGB phases, such as 3-fluoro-4(1-methylheptyloxy) $4^{\prime}-\left(4^{\prime \prime}\right.$-alkoxy-2 $2^{\prime \prime}, 3^{\prime \prime}$ difluorobenzyoyloxy)tolane $\left(n F_{2} B T F O_{1} M_{7}\right)$ [11] which could, in principle, measure the anchoring strength of the capillary wall. Throughout we have neglected the dynamics of the formation of screw dislocations from an undefected sample. The nucleation problem could be avoided experimentally through annealing. It would be an interesting extension of this work, however, to understand defect formation. This task would be considerably easier and tremendously more reliable with some experimental input.

It is a pleasure to acknowledge stimulating discussions with T.C. Lubensky, P.L. Taylor and S. Žumer. The authors acknowledge the hospitality of the Aspen Center for Theoretical Physics, where some of this work was done. RDK was supported by NSF Grants DMR9423114 and DMR91-22645, and TRP by NSF Grant DMR93-50227. 


\section{References}

[1] See, for instance, P.M. Chaikin and T.C. Lubensky, Principles of Condensed Matter Physics, (Cambridge University Press, Cambridge, 1995).

[2] S.R. Renn and T.C. Lubensky, Phys. Rev. A 38 (1988) 2132; 41 (1990) 4392.

[3] J. Goodby, M.A. Waugh, S.M. Stein. R. Pindak, and J.S. Patel, Nature 337 (1988) 449; J. Am. Chem. Soc. 111 (1989) 8119; G. Strajer, R. Pindak, M.A. Waugh, J.W. Goodby, and J.S. Patel, Phys. Rev. Lett. 64 (1990) 13; K.J. Ihn, J.A.N. Zasadzinski, R. Pindak, A.J. Slaney, and J. Goodby, Science 258 (1992) 275.

[4] S. Kralj and S. Žumer, Phys. Rev. E. 51 (1995) 366.

[5] M. Ambrožič and S. Žumer, Phys. Rev. E 54 (1996) 5187.

[6] P.G. de Gennes, Solid State Commun. 14, 997 (1973).

[7] P.G. de Gennes and J. Prost, The Physics of Liquid Crystals, Second Edition, Chap. VII (Oxford University Press, New York, 1993).

[8] A. Sugimura, G.R. Luckhurst and O.-Y. Zhong-can, Phys. Rev. E 52 (1995) 681.

[9] For the case of hexatic membranes, this was studied in S. Seung and D.R. Nelson, Phys. Rev. A 38 (1989) 1005; S. Seung, Ph.D. Thesis, Harvard University (1990).

[10] G.P. Crawford, D.W. Allender, J.W. Doane, M. Vilfan, and I. Vilfan, Phys. Rev. A 44 (1991) 2570.

[11] L. Navailles, C.W. Garland and H.T. Nguyen, J. Phys. II France 6 (1996) 1243. 


\section{Figure Captions}

Fig. 1. Screw dislocation in a capillary geometry. The dark center line is the screw dislocation.

Fig. 2. Reduced critical field $\bar{H}$ vs. reduced capillary radius $\bar{R}$ for $\bar{W}=.30, .54, .96,1.7,3.0$. 


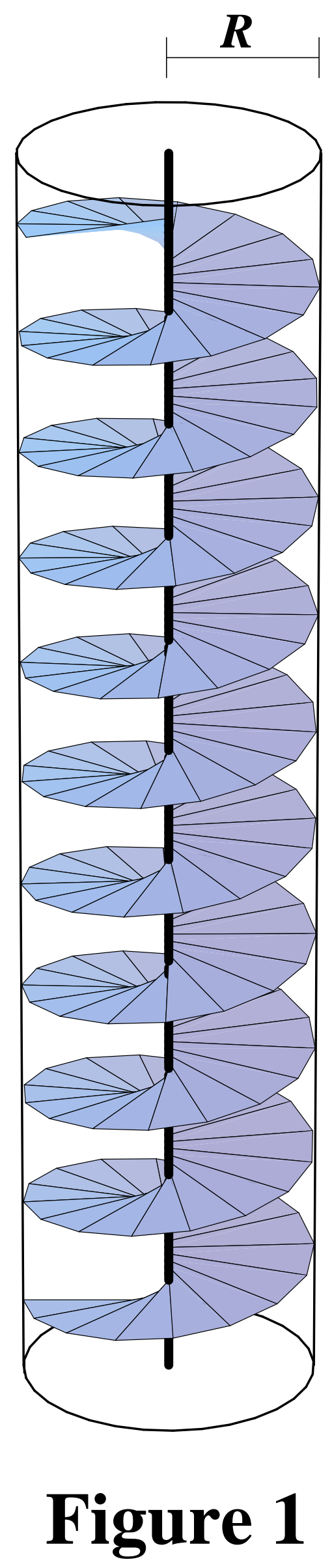




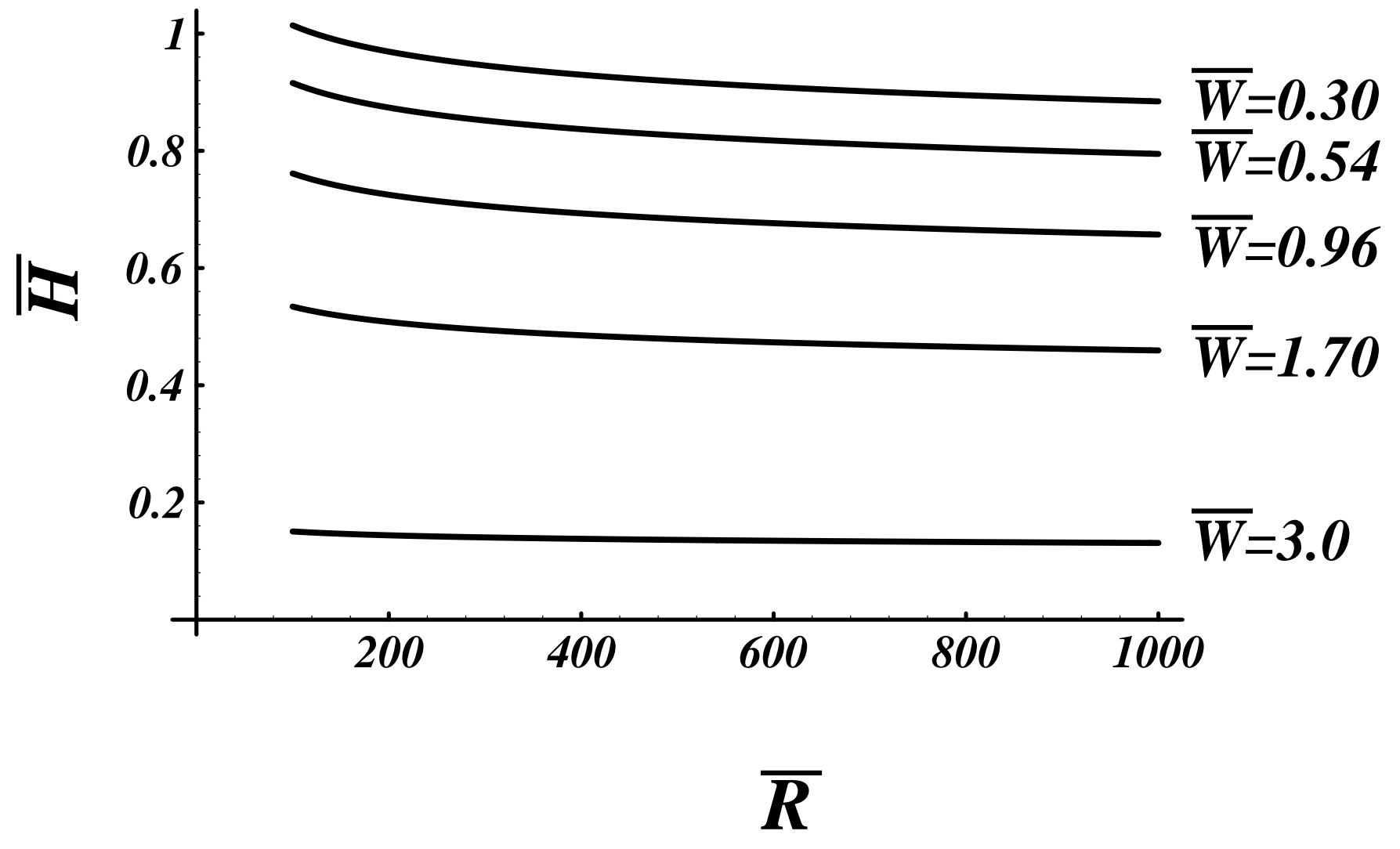

Figure 2 